\title{
Les comités de marine de la Législative et de la Convention, au cœur des rapports entre pouvoir exécutif et législatif
}

\section{Olivier Aranda}

\section{(2) OpenEdition Journals \\ Édition électronique \\ URL : http://journals.openedition.org//rf/3671 \\ DOI : 10.4000/Irf.3671 \\ ISSN : 2105-2557 \\ Éditeur \\ IHMC - Institut d'histoire moderne et contemporaine (UMR 8066) \\ Référence électronique \\ Olivier Aranda, «Les comités de marine de la Législative et de la Convention, au cœur des rapports entre pouvoir exécutif et législatif », La Révolution française [En ligne], 17 | 2020, mis en ligne le 24 février 2020, consulté le 20 avril 2020. URL : http://journals.openedition.org//rf/3671 ; DOI : https:// doi.org/10.4000/Irf.3671}

Ce document a été généré automatiquement le 20 avril 2020.

(c) La Révolution française 


\title{
Les comités de marine de la Législative et de la Convention, au cœur des rapports entre pouvoir exécutif et législatif
}

\author{
Olivier Aranda
}

1 Dans son manuel d'histoire maritime du début $\mathrm{du} \mathrm{xx}^{\mathrm{e}}$ siècle, Joannès Tramond décrit en ces termes les rapports entre assemblée et ministre en ce qui concerne la marine, pendant toute la durée de l'an II : «Dalbarade, type d'officier politicien, timoré, hésitant [...] accepta facilement la nullité où le réduisit le Comité de la marine, délégué par la Convention à la surveillance et la direction du département: le jour où l'on supprima le ministère (1794) ce fut à peine si l'on s'aperçut qu'il y avait quelque chose de changé. Quant au Comité, composé de gens incompétents, [...] il ne fut jamais en état d'exercer une influence sérieuse sur la vie de notre marine ${ }^{1}$.» Le Comité de marine de la Convention ne trouve pas plus grâce aux yeux d'Édouard Chevalier, dans son Histoire de la marine française sous la première République, publiée à la fin du XIX ${ }^{e}$ siècle. Lorsque le Comité, à l'automne 1794, se dote d'une commission consultative, l'auteur lui fait un sort de la manière suivante : « Il est inutile d'appeler l'attention sur la composition de cette commission, dans laquelle les officiers de marine, c'est-à-dire l'élément véritablement important, étaient à peine représentés ${ }^{2}$. »

2 L'historiographie traditionnelle de la marine française a vu, sans surprise, dans les comités de marine révolutionnaires, des rassemblements d'individus issus d'une France terrienne aveugle aux réalités maritimes et militaires, mais aussi responsables d'une surveillance tatillonne de l'exécutif. Malgré ce dédain, la seule présence de la question du Comité de marine dans des ouvrages quasi-exclusivement consacrés à une histoire purement opérationnelle $d u$ fait naval doit interroger. De fait, plus récemment, l'histoire politique de la marine révolutionnaire a suscité quelque intérêt, avec un article de Norman Hampson sur le Comité de marine de la constituante, paru en $1959^{3}$, en même temps que sa thèse sur la mobilisation de la flotte en l'an $\mathrm{II}^{4}$ et l'ouvrage de 
grande qualité de William Cormack, Revolution and Political Conflict in the French Navy ${ }^{5}$, même si ce dernier ne traite que très peu des différents comités de marine. Plus récemment encore, Thibaut Poirot a brillamment éclairé les enjeux de politique partisane sous-jacents dans les décisions maritimes de la Législative ${ }^{6}$. Dans le désert de l'historiographie de la marine révolutionnaire, nous avons là les preuves d'un intérêt certain, en particulier de la part des auteurs anglo-saxons.

3 En effet, loin d'être confinée à des simples questions techniques, la marine se situe au cœur des évolutions politiques et sociales à l'œuvre sous la Révolution. La grande question sous la Constituante est ainsi celle du privilège nobiliaire et de l'accès au corps des officiers de marine, transformant le Comité de marine en une arène politique majeure, comme l'a parfaitement montré Norman Hampson. Sous la Législative, le champ de bataille se déplace : la question brûlante devient celle de l'émigration des officiers, qui met le Comité de marine à la pointe du combat contre l'exécutif royal. L'étude du Comité de marine de la Législative se doit d'être articulée à celle de la Convention, afin de bien cerner le flux et reflux de ces rapports entre pouvoirs exécutif et législatif, rapports parfois conflictuels, le plus souvent constructifs.

Ces relations sont dramatisées par la guerre : la recherche de l'efficacité militaire pousse alors le Comité de marine à se faire le suppléant du pouvoir exécutif et, en même temps, à le mettre sous tutelle. On n'a pas assez dit en effet, et nous reviendrons sur ce point en détail, que c'est au nom des impératifs maritimes que Kersaint, figure majeure du Comité de marine de la Convention, propose la création du Comité de défense générale, décision extrêmement lourde de conséquences par la modification profonde des rapports entre exécutif et législatif qu'elle induit, encore accentuée à partir de la transformation du Comité de défense générale en Comité de salut public. Avec la Convention thermidorienne, le Comité de marine retrouve un rapport plus apaisé au pouvoir exécutif. C'est la période la plus productive, en termes législatifs, de ce Comité, et pourtant paradoxalement la moins étudiée.

D'abord premier opposant puis premier adjuvant à l'exécutif, le Comité de marine tient un rôle clé dans l'évolution des rapports entre pouvoirs, ce qui nous amène à une deuxième affirmation qui découle de la première: les assemblées révolutionnaires n'étaient pas aussi indifférentes au fait maritime que toute une historiographie traditionnelle a bien voulu le croire, et ce, justement, grâce à cette institution du Comité de marine.

6 Les sources pour l'étude du Comité de marine témoignent de son rôle complexe, à la lisière entre exécutif et législatif. La sous-série consacrée à la question aux Archives nationales est très peu utilisable, car elle ne contient presque que des bordereaux de contrôle des pièces envoyées par le ministère ${ }^{7}$. On trouve les procès-verbaux des séances du comité dans la série des registres du Comité de la marine, qui donne une vision assez édulcorée des séances ${ }^{8}$. Les Archives parlementaires restent ainsi le meilleur outil à la disposition de l'historien pour saisir son activité, auxquelles il faut ajouter les traces très intéressantes que ce comité a laissées de ses rapports avec l'exécutif dans la correspondance des bureaux du ministère de la Marine, notamment celle entre le ministre et le Comité de marine et le Comité de défense générale, qui représentent un volume considérable de documentation'. On dispose aussi d'une source précieuse avec les mémoires de Bertrand de Molleville, ministre de la marine en 1791, qui décrit minutieusement son conflit plus ou moins ouvert avec le Comité de marine de la Législative ${ }^{10}$. 


\section{Le Comité de marine de la Législative à la tête de l'affrontement avec l'exécutif royal}

7 Si le Comité de marine se retrouve rapidement à la tête de l'affrontement avec l'exécutif royal, ce n'est pas seulement du fait de considérations liées à l'organisation des pouvoirs, c'est aussi le fait de personnalités, comme celle de Bertrand de Molleville, dont les rapports avec le Comité prennent rapidement un tour venimeux. L'importance de cette personnalité nous pousse à traiter la question d'une manière largement chronologique : le 15 mars 1791, date du départ de Bertrand de Molleville et d'une grande revue des officiers de marine, marque une rupture, soulignée encore par deux renouvellements du Comité de marine les 5 mars et 14 avril 1791.

\section{Un Comité sans orientation a priori ?}

Pourtant, l'établissement du Comité de marine ne semble pas avoir été gros d'une opposition immédiate avec le ministre et le pouvoir exécutif en général. Il y avait d'autant moins de raisons à cela que l'Assemblée constituante avait, les 15 mai et 21 septembre 1791, abouti à une réorganisation générale de la marine, après un débat acharné ${ }^{11}$. Elle consacrait un renforcement du pouvoir civil dans la gestion des ports, l'armement et le mouvement des navires. Elle permettait aussi une ouverture très limitée du corps des officiers aux officiers dits «bleus ", généralement des capitaines marchands ayant obtenu une commission temporaire pendant la guerre d'indépendance américaine ${ }^{12}$. L'égalité devant la loi étant reconnue, la fin du grand corps était néanmoins actée pour l'avenir. Il restait cependant des sujets à traiter, comme celui de l'artillerie de marine, mis en avant dès le 9 octobre par Cavellier comme principal argument en faveur de la création du Comité : «Je finis en réclamant la priorité pour l'établissement du Comité de marine, afin d'organiser les canonniers matelots. Ce corps mérite qu'on s'en occupe par l'importance du service qu'il remplit ${ }^{13}$.» Le Comité de marine est donc établi le 14 octobre 1791 comme « Comité des lois et règlements de la marine ", ce qui semble le cantonner au rôle de laboratoire de la loi, sans empiètement ni contrôle de la sphère exécutive ${ }^{14}$. Certes, avant même l'élection du Comité le 25 octobre, certains députés avaient soulevé les problèmes à venir concernant la marine: Brissot lui-même l'avait incluse dans la question déjà soulevée de la répartition des pouvoirs exécutif et législatif, en réponse au député Ramond: "Il ne veut pas de comité diplomatique, militaire et de marine, parce que suivant lui, le roi a une initiative absolue sur tous ces objets [...] Où M. Ramond a-t-il vu que le roi avait une initiative absolue sur la diplomatie, la marine et le militaire ${ }^{15}$ ?» Par ailleurs, le député Chabot avait posé la question du versement de leurs salaires aux officiers navals absentéistes. Néanmoins, aucun de ceux-ci n'est élu au Comité de marine ${ }^{16}$. En voici la composition, après les désistements du 8 novembre ${ }^{17}$ :

\begin{tabular}{|l|l|l|}
\hline Député & Département & Profession \\
\hline Forfait & Seine Inférieure & Ingénieur constructeur de la marine \\
\hline Aubert-Dubayet & Isère & Officier de l'armée \\
\hline
\end{tabular}




\begin{tabular}{|c|c|c|}
\hline Niou & Charente & Ingénieur constructeur de la marine \\
\hline Dumoustier & $\begin{array}{l}\text { Charente } \\
\text { Inférieure }\end{array}$ & Négociant \\
\hline Cavellier & Finistère & Administrateur de la marine \\
\hline Roujoux & Finistère & Avocat \\
\hline Morand & Côtes du nord & Avocat \\
\hline Rouyer & Hérault & Officier de marine «bleu », maire de Béziers \\
\hline Grégoire aîné & Seine Inférieure & Négociant \\
\hline $\begin{array}{l}\text { Michel de la } \\
\text { Morvonnais }\end{array}$ & Ille-et-Vilaine & Avocat \\
\hline Théodore Lameth & Jura & Officier de marine puis de l'armée \\
\hline Granet (de Toulon) & Var & Avocat \\
\hline Malassis & Finistère & Imprimeur \\
\hline Brunck & Bas-Rhin & Officier de l'armée \\
\hline Coppens & Nord & Procureur du roi près de l'amirauté de Dunkerque \\
\hline Sers & Gironde & Négociant \\
\hline Seranne & Hérault & Négociant \\
\hline Christinat & Seine Inférieure & Négociant \\
\hline Granet (de Marseille) & Bouches du Rhône & Tonnelier et administrateur du département \\
\hline Letourneur & Manche & $\begin{array}{l}\text { Capitaine de génie aux travaux du port de } \\
\text { Cherbourg }\end{array}$ \\
\hline Dufrexou & Loire Inférieure & Avocat, maire de Saint Nazaire \\
\hline Emmery & Nord & Négociant \\
\hline Lafont-Ladebat & Gironde & Négociant \\
\hline
\end{tabular}

9 La composition de ce premier Comité de la Législative appelle plusieurs remarques. Tout d'abord, il comprend exclusivement des élus de départements maritimes, à l'exception de trois militaires: Théodore Lameth, Brunck et Aubert-Dubayet. La répartition entre Méditerranée et Océan est équilibrée, surtout du fait du poids de Granet de Toulon, en permanence président ou vice-président du Comité. Celui-ci n'est donc pas a priori déconnecté des réalités navales, mais sa composition sociale donne à voir des profils maritimes bien particuliers. On ne compte qu'un officier de marine, et il 
s'agit d'un cas particulier: Rouyer a été officier d'appoint pendant la guerre américaine. À l'inverse, les civils de la marine sont bien représentés, avec deux ingénieurs constructeurs : Forfait, le plus notable, figure montante de la construction navale et auteur d'un remarqué Traité élémentaire de la mâture des vaisseaux en 1788, ainsi qu'un administrateur de la marine, Cavellier, qui se trouve par la suite à la pointe du combat contre Molleville. Au vu des rivalités dans les attributions entre militaires et civils dans les ports, ce point n'est pas anodin. La présence très forte de négociants des villes portuaires est importante aussi en ce qu'elle concerne des individus directement impliqués dans les relations avec Saint-Domingue, et a priori favorables à l'extension du recrutement des officiers de marine dans les rangs des capitaines de commerce. Les équilibres partisans dans le Comité sont fluctuants et perturbés par les relations avec l'exécutif, ainsi que par l'aspect technique de certains sujets discutés, qui fait passer les appartenances idéologiques au second plan. Il existe néanmoins des pôles clairs: Rouyer, Niou et Sers sont jacobins ; Théodore Lameth est inscrit aux Feuillants, ainsi que Brunck et Coppens, qui appartiennent au côté droit, mais aussi Forfait, bien que de manière plus ambiguë.

Mais ces clivages ne se décantent qu'avec le temps, à mesure que s'accroît l'opposition au ministre. Dans un premier temps, la concorde entre pouvoirs exécutif et législatif semble possible, en raison notamment de l'unité nécessitée par la crise du moment : la révolte des esclaves à Saint-Domingue. Le 27 octobre, lorsque l'on apprend la nouvelle, Viénot-Vaublanc enjoint le Comité à " conférer avec le ministre ${ }^{18}$ ». Le Comité semble faire preuve de bonne volonté dans ses rapports avec celui-ci, se rendant auprès de lui : " Les comités colonial et de marine réunis [...] ont cru qu'ils devaient envoyer quatre de leurs membres chez le ministre pour connaître les forces de la colonie ${ }^{19}$.» Cet acte lui est d'ailleurs reproché, comme par le député Girardin : «II ne faut pas que les comités soient les intermédiaires entre l'Assemblée et les ministres, et je demande que les comités colonial et de la marine réunis, soient sévèrement réprimandés pour avoir été prendre ces renseignement ${ }^{20}$. » En effet, le Comité de marine se réunit régulièrement à cette date avec le Comité des colonies. Au nom de l'importance des sujets discutés, le Comité de marine en vient même à défendre les envois de troupes de l'exécutif, en dépit de l'opposition des tribunes et du côté gauche de l'Assemblée. Ainsi, le 6 novembre 1791, Forfait fait un rapport au nom du Comité de marine sous la forme d'une injonction au pouvoir exécutif afin qu'il agisse avec énergie dans les secours à apporter à Saint-Domingue ${ }^{21}$. La réaction de la gauche est immédiate: Merlin de Thionville craint qu'on veuille éloigner inutilement l'armée; Lecointre pointe les problèmes de rapports de pouvoirs : "si vous invitez le pouvoir exécutif, si vous le provoquez à envoyer des secours, par là vous détruisez la responsabilité du ministre ${ }^{22}$. » Rouyer, pourtant plutôt du bord politique de Lecointre, vient au secours de Forfait en s'écriant : « Eh ! que m'importe la responsabilité si nous sauvons les colonies! II faut de nouveaux secours, et le Corps législatif a bien le droit d'exciter le zèle du pouvoir

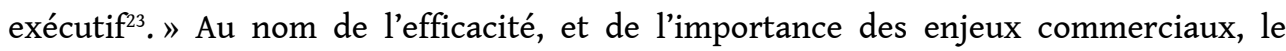
Comité de marine semble donc nouer une alliance de circonstance avec le ministère. Pourtant, à partir de cette position, il évolue rapidement pour devenir le fer de lance du combat contre l'exécutif royal. 


\section{La personnalité de Molleville, déclencheur de l'antagonisme entre exécutif et Comité de marine}

11 Cette transformation tient beaucoup à la personnalité de Bertrand de Molleville, le ministre de la Marine de l'époque. Ancien intendant de Bretagne, royaliste intransigeant, celui-ci exprime clairement dans ses mémoires qu'il a immédiatement choisi l'affrontement avec l'Assemblée, notamment en refusant catégoriquement de se rendre aux réunions du Comité de marine, alors que ses collègues consentent à se rendre auprès des différents comités, après diverses manœuvres dilatoires. Ce refus du ministre de se déplacer auprès du Comité explique aussi le fait que ce dernier ait dû lui envoyer une délégation pour conférer avec lui à propos des troubles à SaintDomingue $^{24}$. Néanmoins, le ministre de la Marine affirme dans ses mémoires s'en être tenu à la lettre de la Constitution : il lutte pour le roi à l'intérieur du champ de celle-ci. Il mène le combat notamment sur des questions de forme: lorsque l'Assemblée demande la signature du roi pour les dépenses qu'il ordonne, le 9 novembre 1791, il refuse, ce qui entraîne une argutie juridique sur les formes prévues par la Constitution ${ }^{25}$. Il lui arrive aussi d'employer le terme de " chevalier ", aboli, jusque dans un discours à l'Assemblée, pour lequel il est immédiatement repris, mais qui contribue à situer politiquement le personnage ${ }^{26}$.

12 Ce sont des rapports en provenance de Brest qui révèlent la profonde fracture entre le ministre et le Comité : le 31 octobre 1791, une pétition des citoyens actifs de la ville demande le remplacement des officiers de marine absents ${ }^{27}$. Plus grave, le 27 novembre, un capitaine envoyé pour commander un des vaisseaux de l'expédition pour Saint-Domingue, Lajaille, est attaqué par la foule à Brest lorsqu'il vient prendre son commandement, étant suspecté "d'incivisme", notamment du fait de son appartenance au grand corps. À la séance de l'Assemblée du 3 décembre, Molleville donne sa version des faits, immédiatement démentie par celle de la municipalité de Brest, qui fait quant à elle porter la responsabilité de l'incident sur le choix du ministre d'un tel officier ${ }^{28}$. L'hostilité au ministre domine dans la salle, au point que le rapport des autorités brestoises reçoit la mention honorable ${ }^{29}$. L'affaire étant renvoyée au Comité de marine, celui-ci se retrouve en pointe dans l'affrontement avec l'exécutif, position qu'il ne quitte plus. Le 5 décembre, lorsque Molleville vient justifier sa nomination du capitaine Lajaille, le député Rouyer, qui avait d'abord semblé disposé à soutenir l'exécutif comme nous l'avons vu, se montre parmi les plus offensifs à l'encontre du ministre ${ }^{30}$. De même, Forfait, qui défendait encore l'exécutif le 23 novembre à propos de la mutinerie du navire l'Embuscade, se tait cette fois-ci. Un autre membre du Comité de marine se met en revanche en avant : Cavellier. Il annonce que le rapport du Comité de marine "prouvera combien on doit peu ajouter de foi à cette prétendue justification ${ }^{31}$ ». Du fait de l'attitude du ministre et de l'exaspération du clivage politique, le Comité de marine est devenu hostile au ministre dans sa majorité. Cette attitude désolidarise désormais nettement le Comité de marine de celui des colonies, qui n'a pas cette animosité envers le ministère, comme le souligne Manuel $\mathrm{Covo}^{32}$. Le Comité de marine est néanmoins lui aussi chahuté : l'Assemblée est alors dans une telle agitation qu'elle refuse la parole à Rouyer.

Molleville réplique le 8 décembre en niant en bloc les accusations. Il va même jusqu'à élaborer un contre-feu en demandant au Comité de marine d'activer la préparation des décrets concernant l'organisation de l'artillerie de marine ${ }^{33}$. Dans ses mémoires, il 
accuse le Comité de ne se consacrer qu'à l'affrontement avec sa personne : il est en tout cas certain que, la marine étant entraînée dans un conflit politique abrupt, les intérêts concrets de celle-ci passent au second plan ${ }^{34}$. À partir de cet incident fondateur, un duel violent s'installe entre Molleville et Cavellier, d'accusations en justifications, parfaitement analysé par William Cormack. Il nous faut simplement insister sur le fait que c'est en qualité de rapporteur du Comité de marine que Cavellier élève ses critiques contre le ministre. C'est notamment le cas le 29 décembre, lorsqu'il attaque Molleville en affirmant que le ministre dissimule l'émigration : selon lui, "le ministre est inexcusable de vouloir pallier leur désobéissance ${ }^{35} »$. Cette accusation a été relayée avec force par une partie de l'historiographie, notamment Lévy-Schneider, qui affirme avec excès : «Bertrand de Molleville [...] encourageait systématiquement l'émigration ${ }^{36}$.» Le débat est compliqué par la définition problématique de celle-ci : Molleville la limite aux officiers absents alors qu'ils sont en service, quand le Comité y inclut les officiers absents de leur quartier maritime, même s'ils ne sont pas employés. Cavellier propose finalement de « déclarer au roi que son ministre de la marine a perdu la confiance de la nation ${ }^{37} »$.

La séance du 13 janvier 1792 voit l'accusation portée de nouveau contre Molleville, mais révèle aussi l'absence d'unité du Comité de marine. Le discours de Cavellier est interrompu par Coppens, qui affirme: «Au comité, les opinions se sont trouvées partagées, et même très partagées. Je demande en conséquence que la discussion soit renvoyée à huitaine (Exclamations.) ", et ce à la grande colère de Rouyer, qui intervient à son tour : « On vient d'avancer un fait faux. J'interpelle M. Sers, notre vice-président, de constater comme le comité a été d'une voix unanime ${ }^{38}$. " Les tensions internes au Comité de marine se retrouvent ainsi étalées en séance et chargées d'un poids politique important, notamment pendant toute la séance du 13. Il ne fait aucun doute que le Comité ait eu une minorité refusant l'attaque contre le ministre de la Marine, ce qui est confirmé par le procès-verbal de la séance, qui paraît avoir été particulièrement houleuse: "Après une longue discussion relative au rapport de Monsieur Cavellier contre le ministre de la Marine, il a été adopté à l'unanimité fort une voix contraire, une douteuse, et trois membres sortis pendant la séance ${ }^{39}$." Dans ses mémoires, le ministre confirme d'ailleurs avoir pu compter sur quelques défenseurs au sein du Comité, en particulier Forfait, qui appuie bruyamment la défense de Molleville du 2 janvier $^{40}$.

15 Le dénouement survient le $1^{\mathrm{er}}$ février 1792 , lorsque Cavellier réitère la proposition de retirer la confiance de l'Assemblée au ministre de la Marine. Le député Grangeneuve surenchérit en demandant à ce qu'on le fasse même décréter d'accusation ${ }^{41}$. Mais c'est bien le Comité de marine qui est l'instrument principal de cet assaut, et les défenseurs de Molleville ne s'y trompent pas, Quatremère de Quincy accusant même le Comité d'être devenu un "bureau d'intrigues ${ }^{42}$ ». La question préalable sur le décret d'accusation donne finalement lieu au premier appel nominal de la Législative, comme le relève William Cormack: c'est dire à quel point le Comité de marine se trouve en première ligne de l'affrontement politique ${ }^{43}$, puisque les appels nominaux sont des révélateurs des rapports de force dans l'Assemblée, dévoilement qui peut alors être transformé en argument en direction de l'opinion publique ${ }^{44}$. Sans surprise, l'appel nominal révèle un Comité divisé, à l'image du vote de la veille au Comité, adopté à la majorité simple ${ }^{45}$. On compte dix votes en faveur de la mise en accusation, parmi lesquels Aubert-Dubayet, dont c'est le seul vote avec le côté gauche, quatre abstentions, et sept votes contre, notamment Forfait et Théodore Lameth. Finalement, la 
délibération sur l'accusation contre le ministre est repoussée par 208 voix contre 196, une chaude alerte pour l'exécutif ${ }^{46}$. Molleville réussit néanmoins à survivre aux accusations du Comité et ne quitte ses fonctions que le 15 mars 1792, et de son propre chef.

Le ministre semble avoir choisi cette date car c'était celle d'une grande revue des officiers de marine dans les ports. Celle-ci ayant révélé l'ampleur catastrophique de l'émigration, le constat n'est plus à débattre, même si Molleville, dans son compte rendu final à l'Assemblée attribue cette situation à «l'impuissance de punir ou de congédier les ouvriers, les maîtres sous-officiers harangueurs séditieux, et protégés par les clubs dont ils forment la majoritée ${ }^{47}$ ». La démission de Molleville correspond de plus à des renouvellements importants du Comité de marine.

\section{La transformation du Comité de marine au printemps 1792}

17 En effet, avant même le départ du ministre le 15, c'est le 5 mars que le Comité a été renouvelé. En voici la composition, ramenée à douze membres ${ }^{48}$ :

\begin{tabular}{|l|l|l|}
\hline Député & Département & Profession \\
\hline Aubert-Dubayet & Isère & Officier de l'armée \\
\hline Dumoustier & Charente Inférieure & Négociant \\
\hline Rouyer & Hérault & Officier de marine « bleu », maire de Béziers \\
\hline Grégoire aîné & Seine Inférieure & Négociant \\
\hline Michel de la Morvonnais & Ille-et-Vilaine & Avocat \\
\hline Théodore Lameth & Jura & Officier de marine puis de l'armée \\
\hline Malassis & Finistère & Imprimeur \\
\hline Brunck & Bas-Rhin & Officier de l'armée \\
\hline Coppens & Nord & Procureur du roi près de l'amirauté de Dunkerque \\
\hline Sers & Gironde & Négociant \\
\hline Seranne & Hérault & Négociant \\
\hline Christinat & Seine Inférieure & Négociant \\
\hline Dufrexou & Poire Inférieure & Avocat, maire de Saint Nazaire \\
\hline Guillois & Avocat \\
\hline
\end{tabular}



n'a pas été reconduit, pas plus que l'autre cheville ouvrière du premier comité : Forfait. On semble avoir voulu ainsi solder la période Molleville. D'ailleurs, les deux nouveaux venus, Lefrancq et Guillois, ne se sont pas exprimés lors du vote le concernant. Par leurs terres d'élection comme par leur profil social, ils ne détonnent pas avec le reste du Comité. Indéniablement, la recomposition correspond à un virage vers le côté droit : les seuls vrais jacobins rescapés sont Rouyer et Sers, alors que de nombreux feuillants ont été reconduits : on y retrouve Coppens, qui a quitté un temps le Comité, Brunck et Michel, tandis que Lefrancq vient se rajouter à ce groupe. Il ne faut pas néanmoins oublier l'aspect théorique de cette composition, certains députés membres ne se rendant pas au Comité, et d'anciens membres continuant en revanche de participer aux séances.

Le Comité de marine aurait pu en rester là, mais, comme d'autres comités de la Législative, sa composition est modifiée un mois plus tard par une adjonction supplémentaire, autrement plus importante, car elle est le fait des Girondins, qui s'introduisent par ce moyen dans le Comité. Elle tient avant tout à la volonté d'y faire rentrer l'ancien suppléant Kersaint, qui a prêté serment le 2 avril. Son adjonction au Comité est proposée dix jours plus tard, par le député jacobin Albitte : «Je demande que le comité de marine soit tenu de présenter un travail sur le régime des classes de la marine et que ce comité soit augmenté de six membres. (Applaudissements) ${ }^{49}$. » Forfait, bien que n'étant plus membre du Comité de marine, questionne immédiatement ce choix, en rappelant d'ailleurs qu'il existe déjà une législation de la Constituante sur les classes. Le but de l'opération ne trompe personne, un député provoquant les rires de l'Assemblée en s'exclamant : «Je demande que M. Kersaint soit nommé au comité de marine ${ }^{50}$. »Ce sont finalement bien six membres qui sont ajoutés au Comité le 14 avril :

\begin{tabular}{|l|l|l|}
\hline Député & Département & Profession \\
\hline Kersaint & Paris & Officier de marine \\
\hline Arena & Corse & Avocat \\
\hline Descrot d'Estrées & Allier & Officier de l'armée \\
\hline Gasparin (démissionnaire) & Bouches du Rhône & Officier de l'armée \\
\hline Leremboure & Basses-Pyrénées & Résident à Saint Domingue \\
\hline Couget & Hautes-Pyrénées & Avocat \\
\hline
\end{tabular}

La physionomie du Comité n'est, en apparence, pas bouleversée par ce changement, même si la composante militaire en sort renforcée. Kersaint est un girondin, mais Descrot d'Estrées appartient quant à lui au côté droit. Ce renouvellement n'en est pas moins fondamental, car Kersaint ne se révèle pas seulement un membre de plus pour le Comité de marine, mais bel et bien un élément moteur de celui-ci : d'une part, parce que ses liens avec Brissot et les autres Girondins lui donnent un poids considérable, d'autant qu'il parle « des heures durant ${ }^{51} »$; d'autre part, parce qu'il est le seul officier de marine du grand corps présent dans le Comité et que, parmi ces officiers, il s'est 
imposé dans la décennie 1780 comme l'un des plus innovants. Lors de l'adoption des plans types pour les vaisseaux de ligne, il est celui qui est chargé de mener un armement expérimental de ces nouveaux vaisseaux, en l'occurrence sur le Léopard, de 1786 à 1788. Les innovations sont de tous types: « caronades [...], nouvelles cuisines, de nouveaux fours et ventilateurs pour la cale et les ponts, [...] un nouveau gouvernail ${ }^{52}$ ». Il transforme aussi le gréement: "Kersaint privilégie la mâture haute et mise sur les voiles de beau temps perroquets et cacatois ${ }^{53}$.» Ce travail est effectué en lien avec... Forfait, qui publie son traité sur les mâtures en 1788. Si l'historiographie traditionnelle a déploré la perte de nombreux officiers savants par l'émigration, il n'a pas été suffisamment souligné que les quelques acteurs réellement à la pointe de l'innovation, Sané, le chevalier de Borda, Kersaint, Forfait, ont tous pris parti pour la Révolution et que deux d'entre eux se retrouvent députés à la Législative. Kersaint est ainsi l'un des plus engagés dans la remise en cause des structures d'Ancien Régime de la marine, ce qui n'est pas étonnant lorsqu'on sait l'inertie qu'il a dû combattre pour tenter d'introduire ses innovations. Son arrivée témoigne d'ailleurs d'une inflexion dans l'action législative du Comité.

\section{Les débats du Comité de la Législative entre technique et politique}

21 L'action du Comité de marine de la Législative fonctionne sur deux modes: d'abord, celui de l'actualité politique, très marqué par l'affrontement avec le ministre, qui ne doit pas faire oublier les premières tendances au renforcement du pouvoir exécutif d'abord manifestées par le Comité, et celui de la législation sur les affaires courantes. Néanmoins, questions techniques et politiques s'entrecroisent fréquemment, y compris sur des sujets comme l'artillerie de marine.

\section{Le travail du Comité de marine de la Législative comme laboratoire de la loi}

L'action du Comité de marine ne se limite pas à des lois importantes et qui engagent les rapports entre exécutif et législatif. Dans un souci d'exhaustivité, nous avons voulu revenir sur l'ensemble des rapports produits par ce comité sous la Législative, de manière à mieux saisir ses dynamiques internes. Voici un tableau de ses membres classés par le nombre de rapports qui leur ont été respectivement confiés :

\begin{tabular}{|l|l|}
\hline Commissaires & Nombre de rapports \\
\hline Granet (de Toulon) & 12 \\
\hline Letourneur & 9 \\
\hline Forfait & 8 \\
\hline Seranne & 7 \\
\hline Anonyme & 5 \\
\hline
\end{tabular}




\begin{tabular}{|l|l|}
\hline Grégoire & 5 \\
\hline Coppens & 4 \\
\hline Kersaint & 3 \\
\hline Cavellier & 3 \\
\hline Rouyer & 2 \\
\hline Michel de la Morvonnais & 2 \\
\hline Malassis & 1 \\
\hline Roujoux & 1 \\
\hline Théodore Lameth & 1 \\
\hline Sers & 1 \\
\hline
\end{tabular}

Ce tableau apporte quelques éclaircissements sur la vie du Comité : Granet, toujours président ou vice-président, est aussi le rapporteur le plus productif, même s'il se cantonne essentiellement aux questions techniques : uniformes, invalides de la marine, récompenses individuelles, sans oublier les conditions de vie des ouvriers des arsenaux. À l'inverse, Cavellier ne rédige que trois rapports, exclusivement à charge contre Molleville. Enfin, on remarque la prégnance du côté droit dans le Comité avec Forfait, mais aussi Coppens, régulièrement rapporteur, surtout dans les premiers temps de l'Assemblée ${ }^{54}$. On peut regrouper ces rapports en différentes thématiques :

\begin{tabular}{|l|l|}
\hline $\begin{array}{l}\text { Rapports faits au nom du Comité de marine par un rapporteur membre de ce } \\
\text { Comité }\end{array}$ & Nombre \\
\hline Personnel de la marine & 33 \\
\hline Travaux des ports (dont Cherbourg) & 5 \\
\hline Cas individuels & 5 \\
\hline Autres & 5 \\
\hline Troubles à Saint Domingue & 3 \\
\hline Commerce et pêches & 3 \\
\hline Fonds du ministère & 3 \\
\hline Armements & 2 \\
\hline Expédition de recherche de La Pérouse & 2 \\
\hline Course & 2 \\
\hline
\end{tabular}


On constate sans surprise la prégnance très forte des questions de personnels: émigration, application de la réforme des classes de la Constituante, formation des officiers... La Législative a dû régler toutes les difficultés soulevées par la réorganisation profonde de la marine par la Constituante, fin 1791, dans un contexte inédit de disparition pure et simple du corps des officiers. Les autres questions sont réduites à la portion congrue, notamment du fait du conflit avec l'exécutif que nous avons déjà relevé. Le débat sur la poursuite des travaux de la rade de Cherbourg parvient à surnager, tout comme le projet d'expédition à la recherche de La Pérouse, qui sont votés sous l'impulsion de Dupetit-Thouars ${ }^{55}$. Il faut aussi souligner l'importance de la question des bois et forêts, le Comité de marine s'opposant à tout projet d'aliénation des forêts nationales, arrêtant "à l'unanimité que le Comité porterait l'avis que la vente des forêts de futaie et de taillis serait très préjudiciable aux biens de l'État, et à la marine française ${ }^{56}$ ». Notons enfin l'action sociale du Comité, menée par Granet de Toulon : celui-ci fait voter l'uniformisation du système de distribution du pain dans les arsenaux. Les ouvriers obtiennent du pain pour eux et leur famille, en retenue sur leur salaire, payés notamment en assignats, ce qui permet une amélioration notable de leur sort. Granet rappelle à cette occasion combien «les ouvriers des ports et arsenaux forment une classe utile et précieuse à la nation ${ }^{57}$ ».

Par cette abondante production législative, le Comité de marine semble donc s'être imposé au centre du jeu politique et des rapports de pouvoirs. Est-ce à dire que l'image des assemblées révolutionnaires comme sourde au fait maritime est une pure invention?

\section{Une place malgré tout limitée pour les questions purement maritimes?}

La question de l'émigration a mis le Comité de marine sur le devant de la scène politique. Ce fait seul peut suffire à nuancer le jugement négatif traditionnellement porté sur les assemblées révolutionnaires en matière maritime. En effet, si ce sujet a pris autant d'importance, c'est par crainte que la France se retrouve privée de marine en cas de conflit, les députés ayant alors pleinement conscience du risque encouru. Néanmoins, il est indéniable que ce sujet a aussi été mis en avant à des fins de tactique politique: les sujets maritimes plus techniques sont moins présents au début de la Législative. C'est après la démission de Molleville que le Comité doit se battre pour obtenir du temps de parole, ce qu'il fait avec pugnacité, secouru notamment par le nouveau ministre, Lacoste, lequel ne rechigne pas à se rendre au Comité contrairement à son prédécesseur ${ }^{58}$. Le 28 mai 1792, il demande que l'on consacre plus de temps aux questions maritimes, avec l'appui de Granet, alors vice-président du Comité ; les rapports du Comité de marine sont ainsi mis constamment à l'ordre du jour ${ }^{59}$. Le ministre appuie sa demande sur la nécessité d'organiser l'artillerie de marine et décrit les arsenaux comme complètement désorganisés. Pourtant, dès le lendemain, une discussion sur les invalides de la marine est brusquement interrompue par le député Delacroix : « Je demande que l'on continue la discussion du rapport de M. Basire sur le point de savoir si la garde du roi sera ou non licenciée. La discussion du projet du 
Comité de marine n'est pas aussi intéressante que celle-là. (Applaudissements dans les

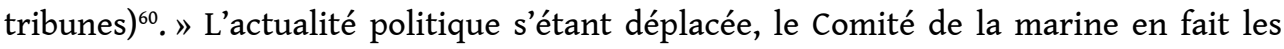
frais. De plus, si les députés sont conscients de l'importance des questions maritimes, les tribunes le sont, sans surprise, nettement moins. Leur pression se fait régulièrement sentir et l'interruption du 29 mai n'est pas isolée. Le 6 avril déjà, on avait coupé la parole au rapporteur du Comité qui traitait de l'arriéré du département de la marine ${ }^{61}$.

Cette indifférence d'une bonne partie des membres et les clivages partisans ont laissé des traces chez certains législateurs, notamment du côté droit, qui en tirent un profond dégoût pour le fonctionnement des assemblées. Ainsi, Forfait, qui ne se représente pas à la Convention, écrit-il en 1802 : « Je regarde la tradition de la confusion des langues arrivée à la construction de la tour de Babel comme une allégorie malheureusement trop expressive de la fatalité attachée à toutes les assemblées délibérantes ${ }^{62}$. C'est $^{\prime}$ ainsi que se forge petit à petit et $a$ posteriori l'idée d'une incompatibilité entre questions maritimes et délibération publique. D'autres membres du Comité ont bien conscience de l'ignorance de la plupart des membres de l'auditoire et recourent à la pédagogie. Ainsi, dès le début de la discussion concernant l'artillerie de marine, Théodore Lameth n'hésite pas à entamer son rapport par une précaution oratoire qui en dit long sur la difficulté de ce Comité à se faire entendre sur ces questions techniques : «Les objets dont je dois vous occuper étant peut-être peu familiers à une partie de membres de cette Assemblée, j'ai cru devoir les présenter avec quelques développements ${ }^{63}$.» La question des régiments d'artillerie et d'infanterie de marine est en effet une des questions importantes traitées par l'Assemblée législative après la démission de Molleville.

\section{Le passage d'un clivage exécutif/législatif à un débat technique}

À partir du départ de Molleville, le Comité ne consacrant plus l'essentiel de son temps à l'affrontement avec l'exécutif, les lois maritimes les plus importantes de la Législative sont votées à partir de cette date sans grande difficulté.

La question la plus importante, celle de l'artillerie de marine, est débattue en séance à partir du 28 mars 1792 et oppose les partisans de ce corps et ceux favorables à sa suppression. Mais c'est aussi sa réorganisation qui divise. Théodore Lameth, rapporteur, propose deux régiments d'artillerie de marine et quatre d'infanterie, estimant pernicieuse leur confusion dans l'organisation précédente (celle des canonniers-matelots de 1786) ${ }^{64}$. Il a existé, durant toute la deuxième moitié du XVIII ${ }^{\mathrm{e}}$ siècle, un débat sur le type d'officiers devant commander ce corps. Lameth les confie aux officiers des troupes de la marine, et non pas aux officiers de marine eux-mêmes, estimant qu'il s'agit de deux métiers différents ${ }^{65}$. De plus, le projet ouvre une partie des postes d'officiers dans ces régiments aux canonniers des classes, qui viennent se rajouter aux régiments en temps de guerre ${ }^{66}$.

30 La question aurait pu en rester à ces considérations strictement techniques si, en seconde lecture, Kersaint n'avait pas proposé une analyse politique du projet, allant même jusqu'à relier la question à celle des rapports entre pouvoirs exécutif et législatif :

31 Je ne considérerai donc le projet de former une artillerie et une infanterie particulières à la marine, que dans ses rapports avec le système de notre nouvelle organisation 
sociale, et les vues générales d'ordre et d'économie qui doivent diriger les représentants d'un peuple libre ${ }^{67}$.

Kersaint craint que ces régiments de marine obéissent aveuglément à l'exécutif : « Sur quel principe, sur quelle nécessité démontrée veut-on vous déterminer à consentir à cette surcharge pour l'État, à cet accroissement du pouvoir militaire dans la main du roi $^{68}$ ? " Il propose donc purement et simplement la suppression de ces régiments. Comme souvent avec Kersaint, derrière un propos iconoclaste et apparemment purement fondé sur la théorie et les principes, se cache un propos très argumenté sur l'organisation de la marine, et fondé sur sa longue expérience maritime, notamment au contact des Anglais. Il propose en fait de confier l'artillerie de marine aux matelots euxmêmes :

Des canonniers réellement matelots, voilà ce qui constituera la force de l'armée navale, et non des canonniers asservis, et qui n'ont que le nom de marins, c'est de l'adresse et non de la science qu'exigent les combats de mer; les canonniers matelots ont des droits sans doute, ils en ont un sacré : car ils sont nécessaires, indispensables; ne les étouffons donc pas sous l'attirail d'une formation de terre ; je parle ici de canonniers citoyens habitant des côtes, véritable force nationale, parce qu'elle est indépendante ${ }^{69}$.

En effet, les régiments proposés ayant beau être des régiments de marine, ceux qui la composent n'en sont pas moins des soldats et non des marins. Les canonniers-matelots eux-mêmes, bien que devant être entraînés en partie à la mer et être familiarisés avec le matelotage comme leur nom l'indique, ne naviguent généralement qu'en rade et sont le plus souvent requis à la surveillance et aux travaux des parcs d'artillerie ${ }^{70}$. Kersaint estime donc qu'ils seront sans doute moins efficaces que des vrais marins navigants en période de paix, et qui ont donc une maîtrise supérieure de l'élément maritime. C'est le cas en Angleterre, où il n'existe pas d'artillerie de marine, mais seulement une infanterie de marine, contrairement à ce qu'affirment certains députés. L'autre intérêt de cette suppression est que les matelots ne seraient pas à la main du pouvoir exécutif. Kersaint n'est néanmoins pas écouté : en troisième lecture, le Comité et ses anciens membres, comme Rouyer et Forfait, s'emploient à combattre son projet sur des aspects techniques plus que politiques - ce qui montre le peu de pertinence des clivages partisans sur cette question - et c'est finalement le projet de Lameth qui est adopté le 14 juin 1792.

Un autre sujet continue d'importer au Comité de marine: celui de l'émigration des officiers. Là encore, Kersaint s'illustre par la radicalité de ses propositions. Refusant un projet qui propose simplement de remplacer les officiers absents par leurs inférieurs en grade, il avance la nécessité de refonder entièrement le corps en y incluant le plus possible de marins, avec, pour principal fondement, l'ancienneté de service sur les vaisseaux de l'État ${ }^{71}$. L'argumentation qu'il déploie est particulièrement intéressante :

C'est pour moi sans doute une nécessité pénible que de me présenter pour la seconde fois à cette tribune, comme contradicteur des idées qui ont obtenu la majorité dans votre Comité de marine ; mais il m'importe que vous sachiez que de 23 personnes qui le composaient, le jour où le plan que je me propose de combattre fut admis, 11 appuyèrent mon opinion de leurs suffrages. Je vous présente cette minorité imposante comme un premier droit à votre attention ${ }^{72}$.

Il est de nouveau fait état en séance des rapports de force au sein du Comité. Mais la capacité d'entraînement de Kersaint vers des réformes radicales ne doit pas être sousestimée. C'est en effet à la toute fin de l'Assemblée législative qu'il parvient à obtenir 
un geste en faveur des officiers "bleus", en s'appuyant sur le contexte d'urgence navale qui pèse sur l'Assemblée au cours de l'été, avec la crainte d'armements anglais ou russes ${ }^{73}$. Ces officiers se voient confirmés à titre permanent le dernier grade temporaire qu'ils ont occupé, abolissant théoriquement les distinctions de corps chez les officiers de marine ${ }^{74}$.

\section{L'extension des rôles du Comité de marine sous la Convention : entre tutelle, relais et administration d'appoint}

Après avoir été en pointe dans l'affrontement avec Molleville au début de la Législative, le Comité de marine se retrouve pleinement acteur de la redéfinition constante des relations entre conseil exécutif, Convention et comités, comme en témoigne son rôle dans la création du Comité de défense générale, qui n'a jusqu'ici pas été suffisamment souligné ${ }^{75}$.

\section{Le Comité de marine, Kersaint et la création du comité de défense générale}

La création d'un Comité de marine trouve un bruyant avocat, sans surprise, dans la personne de Kersaint, fraîchement réélu à la Convention. Dès le 27 septembre, il réclame la reconduction de ce Comité dans un discours qui fait figure de programme :

J'appelle l'attention de la Convention nationale sur l'état actuel de la marine française. Le patriotisme est une sorte de tâche pour la plupart des officiers de cette arme et la Révolution n'est pas encore faite dans l'empire maritime. Ce n'est point la faute du ministre actuel ; mais bien celle des anciens bureaux du ministère qui formaient l'arrière-garde de l'aristocratie. De grands événements se préparent sur terre et sur mer pour le printemps prochain et il est instant que la Convention s'occupe d'organiser nos moyens. II ne tient qu'à nous de recréer cet hiver un armement le plus formidable, si nous savons profiter du temps et des ressources de la République. Je propose la création d'un comité de marine et d'un comité des colonies ${ }^{76}$.

41 Kersaint annonce ici plusieurs choses: la nécessité de poursuivre la réforme du personnel de la marine, mais aussi son hostilité aux bureaux du ministère de la Marine. Le rôle de ces derniers est en effet considérable, du fait du caractère technique de cette institution, où le ministre se contente généralement d'approuver ou non les mesures proposées par ses chefs de bureaux. De plus, Kersaint anticipe un accroissement des tensions en cours avec les nations maritimes, proposant déjà de se préparer à une éventuelle campagne navale pour l'été 1793. Enfin, il met délibérément en parallèle marine et colonies, ce qui anticipe la fusion de ces deux comités en juin 1793. Les questions maritimes s'annoncent donc d'emblée comme une priorité pour la nouvelle législature.

La création du Comité est décidée le 2 octobre 1792, avec un effectif de dix-huit membres $^{77}$. Le 16 octobre, sont élus les conventionnels suivants :

\begin{tabular}{|l|l|}
\hline Membres & Suppléants \\
\hline
\end{tabular}




\begin{tabular}{|l|l|}
\hline Rouyer & Granet (de Marseille) \\
\hline Kersaint & Topsent \\
\hline Niou & Castilhon \\
\hline Defermon & Giraud (de la Rochelle) \\
\hline Faure (de la Seine inférieure) & Guilhermin \\
\hline Bréard & Jeanbon Saint-André \\
\hline Marec & Lefebvre de Chailly \\
\hline Antiboul & Chaumont \\
\hline Taveau & Pocholle \\
\hline Michel (de Lorient) & \\
\hline Sauvé & \\
\hline Maurel & \\
\hline Blad (de Brest) & \\
\hline Rochegude & \\
\hline Philippe Égalité & \\
\hline Ribet & \\
\hline Gaudin & \\
\hline Daubermesnil & \\
\hline
\end{tabular}

Selon l'Almanach national de 1793, le rôle du Comité de marine de la Convention se borne à «préparer les travaux de la Convention sur tout ce qui peut être relatif à l'administration de la marine ", une définition assez restreinte, mais l'apparition du mot "administration" n'est peut-être pas sans motif ${ }^{78}$. Ce sont les Girondins qui dominent ce Comité, les seuls « vrais » Montagnards étant Bréard et Niou. Néanmoins, du fait des remplacements, la composition du Comité évolue, avec notamment l'intégration de Jeanbon Saint-André à partir du 19 novembre ${ }^{79}$.

44 C'est en décembre 1792 que l'activité du Comité prend véritablement de l'ampleur, lorsque les tensions diplomatiques avec la Grande-Bretagne imposent une action résolue dans le domaine maritime. À la suite du fameux rapport du ministre des Affaires étrangères du 31 décembre sur la détérioration des relations avec l'Angleterre, il est décidé que les effectifs des comités concernés, notamment celui de marine, seront complétés, à charge pour le Comité de marine, en même temps que le Comité diplomatique, de présenter un rapport sur la question ${ }^{80}$. Dès le lendemain, Kersaint en profite pour proposer la création du Comité de défense générale. Sans nier bien sûr le 
cadre plus large de cette création, il s'agit ici de montrer à quel point cette innovation se fait au nom de la marine et de ses contraintes propres. En effet, si seul le dernier article est immédiatement voté par la Convention, on ne peut évacuer les autres points avancés par Kersaint dans son discours pour justifier cette proposition. Ce dernier ne traite pas de rapports de pouvoir ou encore d'administration, mais exclusivement de stratégie navale. Kersaint propose en fait un véritable plan de campagne contre la Grande-Bretagne, qui mélange vain espoir de solidarité révolutionnaire et solide analyse stratégique :

Le premier coup de canon tiré sur mer, vous imposera le devoir d'affranchir la Hollande, l'Espagne et l'Amérique; entreprise qui n'est point au-dessus de votre courage et de vos moyens, parce que vous avez pour auxiliaires, un intérêt évident d'une partie de ces nations, et que leur vœu secret vous y appelle ${ }^{81}$.

Il s'agit de retrouver l'alliance des nations maritimes qui a permis de vaincre durant la révolution américaine, et qui est indispensable à un nouveau succès, la France ne pouvant seule faire face à la puissance maritime britannique. Kersaint anticipe un retournement d'alliance de l'Espagne et de la Hollande, qui finit par se produire, dans des circonstances qui ne sont néanmoins pas celles qu'il imagine. Il insiste aussi sur les espoirs, terriblement déçus, fondés sur les États-Unis par de nombreux marins français. Kersaint souligne enfin la nécessité d'une invasion directe de l'Angleterre en cas de conflit, espérant «l'alliance des faubourgs Saint-Antoine et de Black Friars ${ }^{82}$ ». Il s'agit là d'une déclinaison du thème de la fraternité entre les peuples, ici à l'échelle des quartiers populaires de Paris et de Londres, qui se révèle illusoire, l'hostilité primant outre-manche.

Quant aux articles du décret proposé, trop souvent éludés, ils proposent un grand programme de développement de la marine :

48 Article I : Le ministre donnera incontinent des ordres dans tous les ports, pour armer trente vaisseaux de guerre et vingt frégates, et pour qu'ils soient pourvus de quatre mois de vivres et de six mois d'eau.

Article II : La construction de vingt-cinq vaisseaux de ligne, cinq de cent canons, six de quatre-vingt, et quatorze de soixante-quatorze, et vingt frégates de quarante canons portant $\mathrm{du} 24$, sera ordonnée, soit dans les ports de guerre, soit dans ceux du commerce ${ }^{83}$.

50 L'article 9, qui propose la création de ce qui devient le Comité de défense générale, est avant tout pensé pour permettre l'application de ce programme naval. La brève discussion qui suit la proposition de voter cet article seul le prouve. Lorsque le député Charlier s'oppose à la création de ce Comité, arguant que « l'établissement d'un Comité de sûreté extérieure énerverait la responsabilité des ministres", Rouyer vole au secours de Kersaint : «Et moi j'appuie, au contraire la motion de vote immédiat. Je sais que 13 vaisseaux de ligne anglais sont prêts à sortir des ports; il faut se tenir prêts à tout événement ${ }^{84}$. »C'est sur des considérations navales que s'appuie Rouyer, y compris pour défendre l'article 9 seul. Notons qu'on retrouve là, presque terme à terme, la discussion de l'automne 1791 sur l'expédition de Saint-Domingue ! Et l'on mesure à quel point ce sont les circonstances qui ont poussé à l'affrontement avec l'exécutif sous la Législative, tant la tendance naturelle dans le domaine naval est à un renforcement des fonctions exécutives. Car si le rôle du Comité de défense générale est de mettre sous tutelle le conseil exécutif, par trop indépendant de la Convention, il s'agit aussi d'une forme paradoxale de renforcement de l'exécutif, au sein de l'Assemblée, très visible 
dans le cadre de la marine ${ }^{85}$. Ainsi, les bureaux de la marine sont immédiatement assaillis de demandes d'informations de la part du Comité de défense générale, notamment le «tableau de l'état de la marine $»^{86}$ qui doit permettre d'échafauder de nouveaux plans de campagne. La création du nouveau Comité apparaît ainsi comme un stimulus essentiel de l'action politique sur l'administration de la marine, avec des directives précises qui s'accumulent, comme l'ordre de construire des frégates dans les ports de commerce afin de pallier le ralentissement du commerce consécutif à la guerre maritime $^{87}$.

51 La composition du Comité de défense générale et de son bureau en renforce encore le penchant naval. Au lieu de trois membres, le Comité de marine y délègue cinq membres - Rochegude, Taveau, Bréard, Rouyer, Marec - en faisant ainsi le plus représenté. Et cela sans compter Kersaint, qui, s'il est envoyé par le Comité diplomatique, n'en reste pas moins un marin. Si les tensions avec l'Angleterre pèsent pour beaucoup dans cette évolution, tout comme la stratégie générale des Girondins, le tableau n'en est pas moins frappant: jamais il n'y eut, et il n'y eut plus jamais autant d'hommes de mer aux affaires en France qu'en ce début d'année 1793, qu'on associe pourtant généralement à l'atonie de la marine française.

Les premières mesures de ce nouveau Comité le confirment : conjointement avec le ministre des Affaires étrangères, le premier ministre convoqué est celui de la marine. Quant à la toute première affaire traitée, il s'agit d'un rapport sur la course, confié à Rouyer ${ }^{88}$ ! C'est donc par l'outil des comités que les hommes de mer sont parvenus à se tailler au sein de la Convention une place sans rapport avec leur poids réel dans l'Assemblée.

\section{Le Comité de marine de la Convention "montagnarde ", adjuvant ou surveillant du pouvoir exécutif?}

La création du Comité de défense générale correspond à un des moments les plus importants pour le Comité de marine, mais il signe aussi en partie son éclipse, puisqu'une partie considérable des compétences du second est alors transférée au premier. Le décret du 13 janvier sur les armements nécessaires pour la campagne de 1793 est ainsi présenté au nom du Comité de défense générale par Kersaint ${ }^{89}$. Néanmoins, quelques jours plus tard seulement, ce dernier démissionne de son mandat de député, affirmant ne plus pouvoir siéger avec des hommes "de sang" comme Marat ${ }^{90}$. Son passage au Comité de marine, à cheval sur la Législative et la Convention, a eu une grande importance, mais la marine perd là l'un de ses meilleurs ambassadeurs à la Convention.

L'entrée en guerre contre la Grande-Bretagne le $1^{\text {er }}$ février 1793 entraîne logiquement des bouleversements dans la sphère maritime. Le 14 février, Barère fait voter une réorganisation du ministère de la Marine, à l'image de celle du ministère de la Guerre qui vient d'avoir lieu : il est ainsi partagé en six divisions ${ }^{91}$. La veille, Guyton-Morveau avait souligné l'urgence de cette réorganisation, Monge ayant manifesté son intention de quitter le ministère le 12 février ${ }^{92}$. Cette réforme a de nécessaires répercussions sur le fonctionnement du Comité, qui se calque désormais sur l'organigramme ministériel, comme le révèle l'article 5 :

Le comité de la marine sera divisé en six sections, chaque section sera composée de cinq membres qui correspondront à chaque section du département de la marine : en 
conséquence, il sera adjoint au comité de marine de nouveaux membres pour compléter le nombre nécessaire à cette nouvelle division ${ }^{93}$.

En alignant l'organisation du Comité sur celle du ministère, la Convention fait le choix de lui donner une fonction quasiment d'administration d'appoint - ce qui l'éloigne donc du simple rôle de «laboratoire des lois ». Il conserve toutefois son rôle d'évaluateur des innovations, fréquemment proposées, comme durant la seule séance du 12 février 1793 où sont suggérés une " machine infiniment utile à la marine » et un " bonnet [...] capable de parer les coups de sabre $\aleph^{94}$. Mais la correspondance entre le Comité de marine et les bureaux conservée dans les archives traite d'affaires très spécifiques, comme le procès de certains capitaines accusés de lâcheté à la bataille de Prairial, notamment Lucadou ${ }^{95}$. Le Comité de marine penche de plus en plus du côté gauche : parmi les six conventionnels nommés en supplément au Comité du fait de la réforme, le 27 février, on compte quatre montagnards: Barras, Soubrany, Julien de Toulouse et Jullien de la Drôme.

57 Le 10 avril 1793, dans le sillage de la trahison de Dumouriez et de la création du Comité de salut public, le ministre Monge est remplacé par Dalbarade, plus proche des Montagnards. La personnalité de celui-ci joue un grand rôle, à l'image de celle de Molleville, dans les rapports entre l'exécutif et le législatif. Professionnel, il se contente principalement d'un rôle de chef d'état-major, donnant des indications générales sur la manière d'employer la flotte, mais à partir de directives politiques du Comité de salut public, à quelques exceptions près. ${ }^{96}$. Cela permet une remarquable stabilité ministérielle, Dalbarade ne quittant son poste qu'à l'été 1795 .

Le Comité de marine, néanmoins, est loin d'être effacé par ces changements : il garde de nombreuses compétences et, surtout, son autonomie. Manuel Covo a brillamment montré dans sa thèse que, durant tout le printemps 1793, le Comité de marine de la Convention s'oppose au Comité de salut public à propos de la définition de la liberté des mers par rapport à la question cruciale de la navigation des neutres ${ }^{97}$. En effet, le Comité de marine défend à ce propos, le 9 mai 1793, par la voix de son rapporteur Antiboul, une conception très restrictive de la liberté des neutres, inspirée de la règle établie par les Britanniques en 1756. Il s'agit de considérer comme de bonne prise les marchandises embarquées sur des navires neutres, ce qui revient à s'arroger un droit de police des mers, la vérification nécessitant fouille et détournement des navires neutres impliqués ${ }^{98}$. Manuel Covo y voit une revendication des corsaires eux-mêmes, qui multiplient ainsi leurs cibles potentielles. Le Comité de salut public s'oppose frontalement à cette conception, le 23 mai 1793 notamment, par l'organe de Barère, qui souligne les dommages potentiels à l'opinion neutre qui résulteraient d'une telle conception restrictive de la liberté des mers. La politique défendue par le Comité de salut public, aiguillonné par Gouverneur Morris, le chargé d'affaires américain à Paris, est celle du traité franco-américain de 1778, fondée sur la formule " free ships make free goods ", ce qui signifie que les biens même ennemis, s'ils sont transportés par des neutres, acquièrent la neutralité de leur transporteur ${ }^{99}$. Mais dans ce conflit de comités, c'est finalement le Comité de marine qui l'emporte, le 27 juillet, et les navires neutres restent susceptibles d'être arraisonnés par les corsaires et la marine française.

Cet épisode appelle plusieurs remarques. Tout d'abord, Manuel Covo souligne la nuance apportée par cet épisode à l'omnipotence supposée du Comité de salut public; d'ailleurs le ministre Dalbarade semble pencher du côté du Comité de marine dans cette affaire, soulignant les rapports complexes entre ministre et comités, qui ne sont pas à 
sens unique, même dans le cas d'un Dalbarade plutôt conciliant ${ }^{100}$. On retrouve de plus, là encore, l'idée d'un Comité de marine comme outil d'influence pour les députés marins et de départements maritimes, ce qui leur confère un poids supérieur à leur nombre réel. La position du Comité de marine est liée à la défense des intérêts des villes corsaires représentées par ses membres, notamment Taveau, qui est en lien avec les corsaires de Honfleur : ceux-ci ont toute raison d'être satisfait de leur représentant ${ }^{101}$. On se situe donc là encore aux antipodes d'une historiographie voyant dans la Convention un organe sourd au domaine maritime.

On peut néanmoins se demander ce qu'il advient de la stratégie maritime générale de la République au milieu de ces tiraillements. En effet, la position juridique défendue par la France en terme de commerce neutre ne peut en théorie être dé-corrélée d'une stratégie précise. S'arroger le droit de police des mers n'a de sens que dans le cadre d'une véritable maitrise de la mer, sinon le risque est grand de s'attirer l'hostilité des neutres dont on a besoin dans le cadre de son propre ravitaillement, sans pouvoir exercer de pression sur celui de l'ennemi, puisque l'on est bloqué dans les ports. Audelà de l'influence des corsaires, ne faut-il pas voir dans cette décision concernant les neutres l'ambition d'une véritable maîtrise de la mer par la République ? L'action vigoureuse de certains représentants en mission dans les ports, comme Jeanbon SaintAndré, peut laisser envisager une telle option ${ }^{102}$.

61 La nouveauté de la fin de l'été 1793 est en effet l'action des envoyés en mission dans les départements maritimes. Leur impact est ici encore plus considérable qu'ailleurs, car le domaine naval est celui, par essence, de la centralité périphérique : l'essentiel se passe dans les ports et non à Paris. Le plus important est Jeanbon Saint-André, lui-même membre du Comité de salut public, qui, depuis le port de Brest, prend des arrêtés qu'il fait ensuite transformer par la Convention en lois générales ${ }^{103}$. En toute discrétion, le 12 septembre 1793, la Convention change l'organisation administrative: les mouvements des ports, confiés aux civils par la Constituante, repassent aux militaires (c'est-à-dire au commandant des armes) ${ }^{104}$. Dans ce schéma, le Comité de marine est réduit à des tâches subalternes de simple adjuvant de l'exécutif. Bien loin de s'attaquer au ministre, le Comité de marine est devenu le défenseur de celui-ci, comme le montre un incident qui survient le 29 janvier 1794, lorsqu'un conventionnel non nommé s'en prend à Dalbarade :

62 Vous avez ordonné que le citoyen Trulé serait nommé capitaine d'un vaisseau de guerre. Eh bien le ministre a donné ordre à Trulé de se rendre au Port la Montagne pour y être employé en qualité d'enseigne non entretenu. Le ministre a cru apparemment que le décret de la Convention était une atteinte portée à ses prérogatives mais sa conduite ne doit pas rester impunie. Je demande le décret d'accusation contre Dalbarade ministre de la marine ${ }^{105}$.

Bourdon de l'Oise surenchérit immédiatement en voyant là une atteinte à la Convention de la part de l'exécutif. Mais Jeanbon Saint-André s'empresse de voler au secours de Dalbarade : " Je fais observer à la Convention qu'on peut être capitaine d'un vaisseau de guerre seulement avec le grade d'enseigne ou non entretenu; ce fait est connu de tous les marins ${ }^{106}$.» On voit là la solidarité qui existe entre exécutif et Comité de marine, mais aussi l'ignorance des réalités navales d'une bonne partie des conventionnels ${ }^{107}$.

64 Le Comité ne voit ses fonctions administratives assorties d'obligations plus étroites de surveillance qu'au lendemain du 9 thermidor. Le 31 août 1794, il s'organise en 
conséquence, avec huit membres consacrés à la surveillance des affaires générales, et quatre aux affaires particulières ${ }^{108}$. Cette réorganisation passe aussi par le renouvellement rapide des membres du Comité, parmi lesquels Barras, en charge des questions d'administration de la marine, et Fouché, qui est affecté quant à lui à la Marine économique, c'est-à-dire civile ${ }^{109}$.

\section{Le Comité thermidorien, le Directoire et la question sensible de l'administration des ports}

La Comité de marine thermidorien a voulu rompre avec le rythme effréné des lois votées pendant l'an II en refaisant du Comité le point central d'élaboration des lois, de manière à éviter les particularismes qui risquaient de réapparaître à travers l'action non concertée des représentants en mission. Le Comité souhaite prendre son temps pour refonder les lois maritimes, proposant un «plan général de législation sur cette partie ${ }^{110} »$. Mais, comme souvent, cette volonté de refondation durable n'aboutit qu'à une étape de plus dans le marathon législatif de la Révolution.

Il y a néanmoins bien un tournant: dans son rapport au nom du Comité de marine du 15 octobre 1794, Boissier affirme que le but de la marine est « surtout [d']établir de fait le principe incontestable de la liberté des mers ", choix qui vise à souligner les atteintes au droit des gens de la Grande-Bretagne, dans une perspective grotienne qui est poursuivie jusque sous l'Empire ${ }^{111}$. Les premiers résultats sont présentés quelques mois plus tard, début juillet 1795, peu après le débarquement des émigrés par les Anglais à Quiberon. Par la voix de Gouly, le Comité thermidorien souligne, le 3 juillet 1795, le danger immédiat posé par la mer, alors que les fronts terrestres s'éloignent : "Aujourd'hui que ces armées victorieuses ont franchi les sommets, jusqu'à ce moment inaccessibles, des Alpes et des Pyrénées, [...] vous devez, sans cesser de porter un œil vigilant sur l'armée de terre, jeter un regard actif et vivifiant sur l'armée navale ${ }^{112}$. La volonté de réforme est affichée dans le style thermidorien : il s'agit de «faire oublier [...] les agitations convulsives d'une révolution qui fut trop longtemps souillée par les horreurs de l'anarchie et du vandalisme ${ }^{113}$. » La veille, Dalbarade, relique de la période précédente, a été remplacé par Redon de Beaupréau à la commission de la marine et des colonies. Encore peu auparavant, à l'imitation des Anglais, un bureau de longitude a été créé et confié aux sommités du moment, comme Borda, Laplace et Bougainville ${ }^{114}$. Les ambitions maritimes sont donc clairement affirmées.

Pour la grande loi de refondation, le Comité de marine en appelle aux bonnes volontés : "Les citoyens instruits de la théorie et de la pratique [...] des arts et des sciences maritimes, et en particulier les sociétés populaires, sont invités à adresser, sous le plus court délai, au Comité de marine et des colonies, leurs vues, plans et projets ${ }^{115}$. » C'est encore dans le but de faciliter son travail que le Comité a aussi recours à une commission consultative. Voici comment Boissier présente la composition de cette commission, a posteriori, lorsqu'il défend son travail au Conseil des Cinq-Cents le 15 janvier 1796 :

Pléville Le Peley, ancien capitaine de vaisseau. Ses rares qualités avaient déterminé le directoire exécutif à l'appeler au ministère de la marine : sa modestie [...] l'a porté à refuser cette place.

Lacrosse, capitaine de vaisseau, ci devant major général de la Marine à Brest, connu par ses talens [sic] militaires et par la reprise de la Martinique.

Boisquesnay, ancien capitaine de vaisseau et de port. 
Gautier, ancien directeur des constructions à Toulon, il a recréé et organisé la marine espagnole.

Vial du Clairbois, ingénieur-constructeur, connu par la part qu'il a eue à la composition de l'article Marine de l'Encyclopédie, et par ses ouvrages élémentaires et classiques sur cette partie.

Doumest-Revest, ingénieur constructeur, à Toulon.

Even, agent maritime à Nantes.

Lefebvre, inspecteur de la marine, à Rochefort.

Jullou, chef civil de la marine, à Brest.

Bidard, armateur et capitaine du commerce, à l'Orient.

Montaut, idem, à Bordeaux.

Pinatel, idem, à Marseille.

Jullian, idem, à Cette.

Menais Robert, armateur à Port-Malo.

Romagnac, idem, à Marseille.

Dewinck, idem, à Dunkerque ${ }^{116}$.

Cette présentation met en valeur, en les plaçant en premier, les militaires de la commission, pourtant peu nombreux. Cela s'explique par le fait que les conventionnels ont voté, avec l'aide de cette commission, la loi refondant la marine, le 25 octobre 1795 (dernier jour de la Convention), qui redonne notamment aux civils une place importante dans la direction et les mouvements des ports. Boissier est donc ici dans un processus de justification. Édouard Chevalier estime cette commission déconnectée des réalités maritimes :

Le comité, chargé de préparer les lois sur la marine, avait cru nécessaire de recourir aux lumières des hommes spéciaux. Il avait, dans ce but, appelé, auprès de lui, une commission consultative. Il est inutile d'appeler l'attention sur la composition de cette commission, dans laquelle les officiers de marine, c'est-à-dire l'élément véritablement important, étaient à peine représentés ${ }^{117}$.

70 Il ne s'agit pas ici que d'éléments anecdotiques liés à l'administration de la marine. En effet, le vote de cette loi à l'extrême-fin de la Convention relance le conflit entre exécutif et législatif pour le Directoire. Dès le 3 décembre 1795, celui-ci fait passer aux Cinq-Cents le message suivant: «[Le Directoire] a reconnu avec regret que cette organisation [...] ne présentait au gouvernement que des entraves, et l'enchaînait (il doit vous le déclarer) dans tous ses moyens d'exécution ${ }^{118}$." Suspendue, la loi des conventionnels n'est finalement appliquée qu'après une résolution du conseil des Anciens du 29 janvier 1796. Entre-temps, les membres réélus du Comité de marine de la Convention, notamment Boissier, ont défendu bec et ongle leur loi aux Conseils... Ces trois mois de vide juridique dans l'intervalle entre deux campagnes navales n'ont sans doute pas été sans effets négatifs sur la marine, à un moment où la situation évolue fondamentalement avec le changement de camp de l'Espagne et de la Hollande. En effet, la campagne navale de l'été 1796 est la première menée conjointement avec l'aide de l'Espagne, modifiant en profondeur la donne stratégique, notamment en Méditerranée.

71 Les questions maritimes ont donc été, depuis la Législative jusqu'au Directoire compris, au cœur du rapport entre les pouvoirs exécutif et législatif. Leur spécificité n'y est pas étrangère: l'administration comme l'emploi de l'outil naval supposent un puissant exécutif, efficace et ambitieux. Si la Législative a d'abord combattu l'exécutif royal et son ministre, cela est en bonne partie lié au problème de l'émigration et à la personnalité même de Molleville. Dès que les tensions navales ont augmenté, le Comité 
de marine s'est retrouvé en première ligne dans la redéfinition des rapports entre exécutif et législatif, avec la création du Comité de défense générale, dont il est l'artisan principal. À rebours de l'historiographie traditionnelle, il faut souligner que c'est justement grâce à l'outil du Comité de marine que les questions maritimes ont bien résisté au choc de leur questionnement au sein d'une assemblée nombreuse et majoritairement terrienne. C'est bien plus tard, notamment avec la bataille d'Aboukir, que la France commence, à tort ou à raison, à désespérer de sa marine.

\section{NOTES}

1. Joannès TRAMOND, Manuel d'histoire maritime de la France, Paris, Challamel aîné, 1916, p. 584.

2. Louis Édouard CHEVALIER, Histoire de la marine française sous la première république, Paris, Hachette, 1886, p. 228.

3. Norman HAMPSON, «The 'Comité de Marine' of the Constituent Assembly ", The Historical Journal, II, 2, Cambridge, Cambridge university Press, 1959, p. 130-148.

4. Norman HAMPSON, La marine de l'an II : mobilisation de la flotte de l'Océan, 1793-1794, Paris, Marcel Rivière, 1959.

5. William S. CORMACK, Revolution and Political Conflict in the French Navy 1789-1794, Cambrige, Cambridge University Press, 2002.

6. Thibaut POIROT, «La marine à la tribune, les députés et la guerre navale pendant la législative ", dans Anne de Mathan, Pierrick Pourchasse et Philippe Jarnoux, La mer, la guerre et les affaires: enjeux et réalités maritimes de la Révolution française, Rennes, PUR, 2017, p. 85-98.

7. Archives nationales (AN dans le reste des notes), sous-série D/XVI.

8. AN, sous-série $\mathrm{D}^{*} / \mathrm{XVI} / 1$ à 5 .

9. Service Historique de la Défense (SHD dans le reste des notes), sous série BB/3, sous-série BB/1, carton 8 notamment.

10. Antoine BeRTRAND DE MOlleville, Private memoirs of A.F. Bertrand de Moleville, Minister of State, 1790-1791, relative to the last year of the reign of Louis the Sixteenth, Boston, J. B. Millet Company, 1909.

11. Norman HAMPSON, "The 'Comité de Marine' of the Constituent Assembly », art. cité, p. 147.

12. Sur cette question des officiers «bleus", par opposition aux rouges du grand corps, voir Jacques AMAN, Les Officiers bleus dans la marine française au XVIII ${ }^{e}$ siecle, Paris, Droz, 1976.

13. Archives parlementaires (AP dans le reste des notes), t. 34, 9 octobre 1791, p. 149.

14. AP, t. 34, 14 octobre 1791, p. 223.

15. AP, t. 34,14 octobre 1791, p. 228.

16. AP, t. 34,9 octobre 1791, p. 173.

17. Edna Hindie LEMAY, Dictionnaire des législateurs 1791-1792, Ferney-Voltaire, Centre international d'étude du XVIII ${ }^{\mathrm{e}}$ siècle, 2007.

18. AP, t. 34, 27 octobre 1791, p. 438.

19. Ibid., p. 455.

20. Ibid., p. 461.

21. AP, t. 34, 6 novembre 1791, p. 661 .

22. Ibid.

23. Ibid.

24. Antoine BERTRAND DE MOLLEVILLE, Private memoirs..., op. cit., p. 222-223. 
25. Ibid., p. 223-224.

26. $A P$, t. 35, 5 décembre 1791, p. 587.

27. AP, t. 34, 31 octobre 1791, p. 533.

28. AP, t. 35, 3 décembre 1791, p. 527-528.

29. Ibid., p. 529.

30. AP, t. 35, 5 décembre 1791, p. 590.

31. Ibid.

32. Manuel covo, "Le Comité des colonies ", La Révolution française [En ligne], 3 | 2012, mis en ligne le 20 décembre 2012, consulté le 19 juin 2019.

33. AP, t. 35, 8 décembre 1791, p. 646.

34. Antoine BERTRAND DE MOLLEVILLE, Private memoirs..., op. cit., p. 259.

35. AP, t. 36, 29 décembre 1791, p. 638.

36. Léon LÉVY-SCHNEIDER, Le conventionnel Jeanbon Saint-André, Paris, F. Alcan, 1901, p. 306.

37. Ibid.

38. AP, t. 37, 13 janvier 1792 , p. 367.

39. AN, D*/XVI/1, fo 12 , séance du 12 janvier 1792.

40. Antoine BERTRAND DE MOLLEVILLE, Private memoirs, op. cit., p. 261.

41. $A P$, t. $38,1^{\text {er }}$ février 1792, p. 80 . Soulignons ici l'emploi politique qui est fait d'une procédure à l'origine réservée au pénal.

42. AP, t. $38,1^{\text {er }}$ février 1792, p. 89.

43. William S. CORMACK, Revolution and Political Conflict in the French Navy 1789-1794, op. cit., p. 138.

44. Anne SIMONIN et Corinne LECHEVANTON-GOMEZ, " L'appel nominal, une technique pour la démocratie extrême (1789-1795) ? ", Annales historiques de la Révolution française, n 357, juilletseptembre 2009, p. 67-101.

45. AN, D*/XVI/1, fo 16 , séance du 30 janvier 1792.

46. AP, t. $38,1^{\text {er }}$ février 1792, p. 92.

47. AP, t. 40, 27 mars 1792, p. 550.

48. AP, t. 39, 5 mars 1792, p. 394.

49. AP, t. 41,12 avril 1792, p. 517.

50. Ibid., p. 517.

51. Edna Hindie LEMAY, Dictionnaire des législateurs 1791-1792, op. cit., t. 2, p. 429.

52. Sylviane LLINARES, Marine, propulsion et technique : l'évolution du système technologique du navire de guerre français au XVIII siecle, Paris, Libr. de l'Inde, 1994, p. 219.

53. Ibid.

54. Forfait est ainsi à l'origine de l'ensemble des rapports sur la révolte de Saint-Domingue.

55. AP, t. 47, 28 juillet 1792, p. 243.

56. AN, D*/XVI/1, fo 14 .

57. AP, t. 37, 2 janvier 1792, p. 4-5.

58. AN, D*/XVI/1, fo 29, séance du 21 mai 1792.

59. AP, t. 44, 28 mai 1792, p. 189.

60. $A P$, t. 44,29 mai 1792, p. 290.

61. AP, t. 41, 6 avril 1792, p. 235.

62. Pierre-Alexandre FORFAIT, Lettres d'un observateur sur la marine, Paris, Clousier, 1802, p. 12.

63. AP, t. 40, 28 mars 1792, p. 598.

64. Ibid., p. 601.

65. Julien DAGET, Les troupes de la marine, 1774-1816, mémoire de maîtrise sous la direction de JeanPierre Poussou, Paris IV, 2001, p. 35.

66. AP, t. 40, 28 mars 1792, p. 601.

67. Ibid. 
68. Ibid.

69. AP, t. 40, 28 mars 1792, p. 602.

70. Julien DAGET, Les troupes de la marine, 1774-1816, op. cit., p. 88.

71. AP, t. 44, 31 mai 1792, p. 386.

72. Ibid., p. 379.

73. Thibaut POIROT, « La marine à la tribune, les députés et la guerre navale pendant la législative », art. cité, p. 95.

74. AP, t. 114,18 septembre 1792, p. 75.

75. On trouvera plus d'éléments sur les affaires courantes traités par le Comité dans Léon LÉvYSCHNEIDER, Le conventionnel Jeanbon Saint-André, op. cit., p. 308-309 notamment.

76. AP, t. 52, 27 septembre 1792 , p. 79.

77. AP, t. 52, 2 octobre 1792, p. 278.

78. Almanach national pour 1793, BNF, 8-LC22-46, p. 117.

79. $\mathrm{AN}, \mathrm{D}^{*} / \mathrm{XVI} / 2$, fo 6 , séance du 19 novembre 1792.

80. AP, t. 56, 31 décembre 1792, p. 102.

81. AP, t. $56,1^{\mathrm{er}}$ janvier 1793 , p. 114

82. Ibid., p. 116.

83. Ibid. Les frégates proposées, très lourdes par leur taille comme par leur calibre d'artillerie, s'imposent dans les années qui suivent, notamment dans la marine américaine. Très ambitieux, le programme de Kersaint est aussi pertinent dans ses choix.

84. AP, t. 56, $1^{\text {er }}$ janvier 1793, p. 117.

85. Virginie MARTIN, La diplomatie en Révolution: structures, agents, pratiques et renseignements diplomatiques, thèse de doctorat sour la direction de Jean-Clément Martin, Paris 1, 2011, 2 vol., t. 2, p. 87.

86. SHD, BB $/ 3 / 35$, fo 265 .

87. SHD, BB $/ 3 / 35$, fo 270 .

88. François-Alphonse AULARD, Recueil des actes du Comité de salut public avec la correspondance officielle des représentants en mission et le registre du Conseil exécutif provisoire, tome 1, Paris, Impr. nationale, 1913, p. 401.

89. AP, t. 57, 13 janvier 1793, p. 38.

90. Armand-Guy Kersaint au président de la Convention nationale, BNF, 8-LB41-2616, p. 1.

91. AP, t. 58, 14 février 1793, p. 524-525.

92. AP, t. 58, 13 février 1793, p. 495.

93. AP, t. 58, 14 février 1793, p. 524.

94. AN, D*/XVI/2, fo 18 , séance du 12 février 1793.

95. AN, D/XVI/3, Lettre de Lucadou du 10 messidor an II (28 juin 1794).

96. Voir notamment son remarquable plan de campagne pour 1793 au SHD, BB/4/14, fo 34-56.

97. Manuel covo, Commerce, empire et révolutions dans le monde atlantique : la colonie de SaintDomingue, entre métropole et États-Unis, thèse de doctorat sour la direction de François Weil, EHESS, 2014, p. 377

98. Ibid., p. 378.

99. Ibid., p. 380.

100. Ibid., p. 381.

101. Ibid., p. 382.

102. Olivier ARANDA, La bataille de Prairial : le Fleurus de la marine républicaine?, Mémoire de M2 sous la direction de Pierre Serna, Paris 1 Panthéon-Sorbonne, 2015, p. 196.

103. Ibid, p. 76.

104. Recueil des lois relatives à la marine et aux colonies. T. 4. Du $1^{\text {er }}$ juillet 1793 au $1^{\text {er }}$ thermidor an II, Bibliothèque Nationale de France, F-42688, p. 91-92 
105. Réimpression de l'ancien Moniteur: Convention nationale, tome XIX, Paris, 1859, p. 340.

106. Ibid., p. 342.

107. Et, de fait, Barère réagit en demandant un grade de capitaine de vaisseau pour le dénommé Trulé, que la Convention lui accorde, malgré les récriminations de Jeanbon Saint-André et de Legendre devant un tel bond en grade.

108. AN, AD/VII/39, 14 fructidor an II (31 août 1794).

109. Ibid.

110. $\mathrm{AN}, \mathrm{AD} / \mathrm{VII} / 39$, Rapport de Boissier, 24 vendémiaire an III (15 octobre 1794).

111. Ibid.

112. AN, AD/VII/39, Rapport de Gouly, 15 messidor an III (3 juillet 1795).

113. Ibid.

114. AN, AD/VII/39, 7 messidor an III (25 juin 1795).

115. $\mathrm{AN}, \mathrm{AD} / \mathrm{VII} / 39$, Rapport de Boissier, 24 vendémiaire an III (16 octobre 1795).

116. $\mathrm{AN}, \mathrm{AD} / \mathrm{VII} / 40$, Justification de Boissier, ancien conventionnel, aux Cinq-Cents, 25 nivôse an IV (15 janvier 1796).

117. Louis Édouard CHEVALIER, Histoire de la marine française sous la première république, op. cit., p. 228.

118. $\mathrm{AN}, \mathrm{AD} / \mathrm{VII} / 40$, Message du directoire exécutif, 12 frimaire an IV (3 décembre 1795).

\section{RÉSUMÉS}

L'historiographie traditionnelle de la marine révolutionnaire a vu dans les assemblées révolutionnaires des organes insignifiants et incompétents. Cette position doit être nuancée par le fait que le Comité de marine se retrouve au cœur des dynamiques politiques de la Législative et de la Convention. C'est notamment le cas en ce qui concerne les relations entre exécutif et législatif : le Comité de marine se trouve, durant la Législative, à la tête de l'affrontement contre l'exécutif royal, à travers la question de l'émigration des officiers de marine, au point de provoquer le premier appel nominal de la législature sur la question. Son importance se renforce encore sous la Convention, où, loin de s'opposer à l'exécutif, le Comité de marine plaide cette fois-ci en faveur de son renforcement. La création du Comité de défense générale le $1^{\text {er }}$ janvier 1793 est proposée par un membre éminent de ce Comité, Kersaint, au nom de la montée des périls maritimes, en premier lieu la guerre imminente avec la Grande-Bretagne.

Les relations entre exécutif et législatif dans le domaine maritime retrouvent un caractère conflictuel sous le Directoire, qui refuse d'appliquer la réorganisation maritime décidée par la Convention thermidorienne, ce qui souligne encore l'importance des questions maritimes à cette période.

Traditional historiography of the revolutionary navy depicts the revolutionary assemblies as insignificant and clueless about naval matters. This position must be balanced by the position of the Navy Committee, at the core of the politics of both Legislative and Convention. It is particularly true concerning the relations between the executive and legislative powers: the Navy Committee leads the fight against the royal executive power during the Legislative, due to the importance of the issue of the emigration of naval officers. This matter, raised by the Naval Committee, triggers the first roll-call vote of this assembly. The Committee gets even more important during the Convention, where it executes a complete reversal of its orientation to 
arguing for a swift reinforcement of executive powers. It is a member of the Navy Committee, putting forward the increased dangers of maritime warfare in the coming months, who proposes the creation of the Committee of General Defence on January the $1^{\text {st }}, 1793$.

Relations between the executive and legislative powers get tense again in the maritime field under the Directory, the latter refusing to apply the laws voted by the late Convention, a fact that underlines again the importance of naval affairs in revolutionary France.

\section{INDEX}

Mots-clés : Marine, Révolution Française, comité de marine, comité de défense générale, Kersaint

Keywords : Naval History, French Revolution, Navy Committee, Committee of General Defence, Kersaint

\section{AUTEUR}

\section{OLIVIER ARANDA}

Doctorant en histoire

IHRF-IHMC

Université Paris 1 Panthéon-Sorbonne 